\title{
Summary of ALSEP Test Loop Solvent Irradiation Testing
}

Fuel Cycle Technology

Prepared for

U.S. Department of Energy Material Recovery and Waste Form Development Campaign Dean Peterman and Lonnie Olson Idaho National Laboratory August 2016 INL/EXT-16-39626 



\section{DISCLAIMER}

This information was prepared as an account of work sponsored by an agency of the U.S. Government. Neither the U.S. Government nor any agency thereof, nor any of their employees, makes any warranty, expressed or implied, or assumes any legal liability or responsibility for the accuracy, completeness, or usefulness, of any information, apparatus, product, or process disclosed, or represents that its use would not infringe privately owned rights. References herein to any specific commercial product, process, or service by trade name, trade mark, manufacturer, or otherwise, does not necessarily constitute or imply its endorsement, recommendation, or favoring by the U.S. Government or any agency thereof. The views and opinions of authors expressed herein do not necessarily state or reflect those of the U.S. Government or any agency thereof. 



\section{CONTENTS}

SUMMARY

Error! Bookmark not defined.

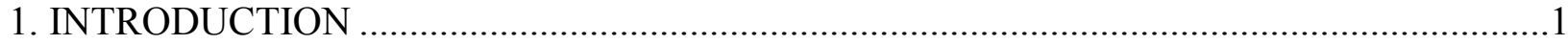

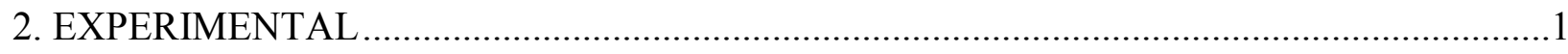

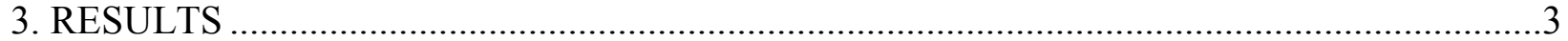

4. CONCLUSIONS 



\section{Summary of ALSEP Test Loop Solvent Irradiation Testing}

\section{INTRODUCTION}

Separating the minor actinide elements (americium and curium) from the fission product lanthanides is an important step in closing the nuclear fuel cycle. Isolating the minor actinides will allow transmuting them to short lived or stable isotopes in fast reactors, thereby reducing the long-term hazard associated with these elements. The Actinide Lanthanide Separation Process (ALSEP) is being developed by the DOENE Material Recovery and Waste Form Development Campaign to accomplish this separation with a single process. To develop a fundamental understanding of the solvent degradation mechanisms for the ALSEP Process, testing of the extraction section of the ALSEP flowsheet was performed in the INL Radiolysis/Hydrolysis Test Loop. This work culminated in the completion of the level two milestone (M2FT-16IN030102021) "Complete ALSEP test loop solvent irradiation test." This report summarizes the testing performed and the impact of radiation on the ALSEP Process performance as a function of dose.

\section{EXPERIMENTAL}

The irradiation source is an MDS Nordion GammaCell 220 Excel self-contained ${ }^{60} \mathrm{Co}$ gamma irradiator. The center-line gamma dose rate in the sample chamber is approximately $5.4 \mathrm{kGy} / \mathrm{hr}$. The solvent irradiation loop is based upon a coil of borosilicate glass tubing ( 0.375 " OD, 0.202 " ID) which is placed in the gamma irradiator sample chamber. The current effective gamma dose rate in the test loop is 3.3 $\mathrm{kGy} / \mathrm{hr}$. During the solvent irradiation, the aqueous and organic phases are mixed using a centrifugal contactor (CINC V-02, USA) with the rotor replaced by a four vane mixing paddle. The organic and aqueous phases used are metered into the mixing region of the contactor at the desired organic to aqueous phase ratio $(\mathrm{O} / \mathrm{A})$. The mixed phases are pumped through the irradiator test loop by a magnetic drive gear pump. The mixed phases flow through the glass coil in the irradiator sample chamber, through an external temperature controlled loop, and return to the inlet of the centrifugal contactor where the phases are mixed and circulated back through the loop. In-line tube mixers (TAH Industries, Inc.) are used to provide additional phase mixing in the test loop. The in-line tube mixers are placed between the outlet of the gear drive pump and the inlet of the external loop. The flowrate of the dispersion is in the range of 1.5 $\mathrm{L} / \mathrm{min}$ in order to maintain turbulent flow and keep the phases dispersed. The radiolysis/hydrolysis test loop is equipped with pressure gauges and thermocouples before and after the gamma irradiator sample chamber. A flow sight glass is used to monitor the extent of mixing in the test loop. The two phases continue to circulate until the desired dose is obtained. A schematic view of the radiolysis/hydrolysis test loop is shown in Figure 1. 


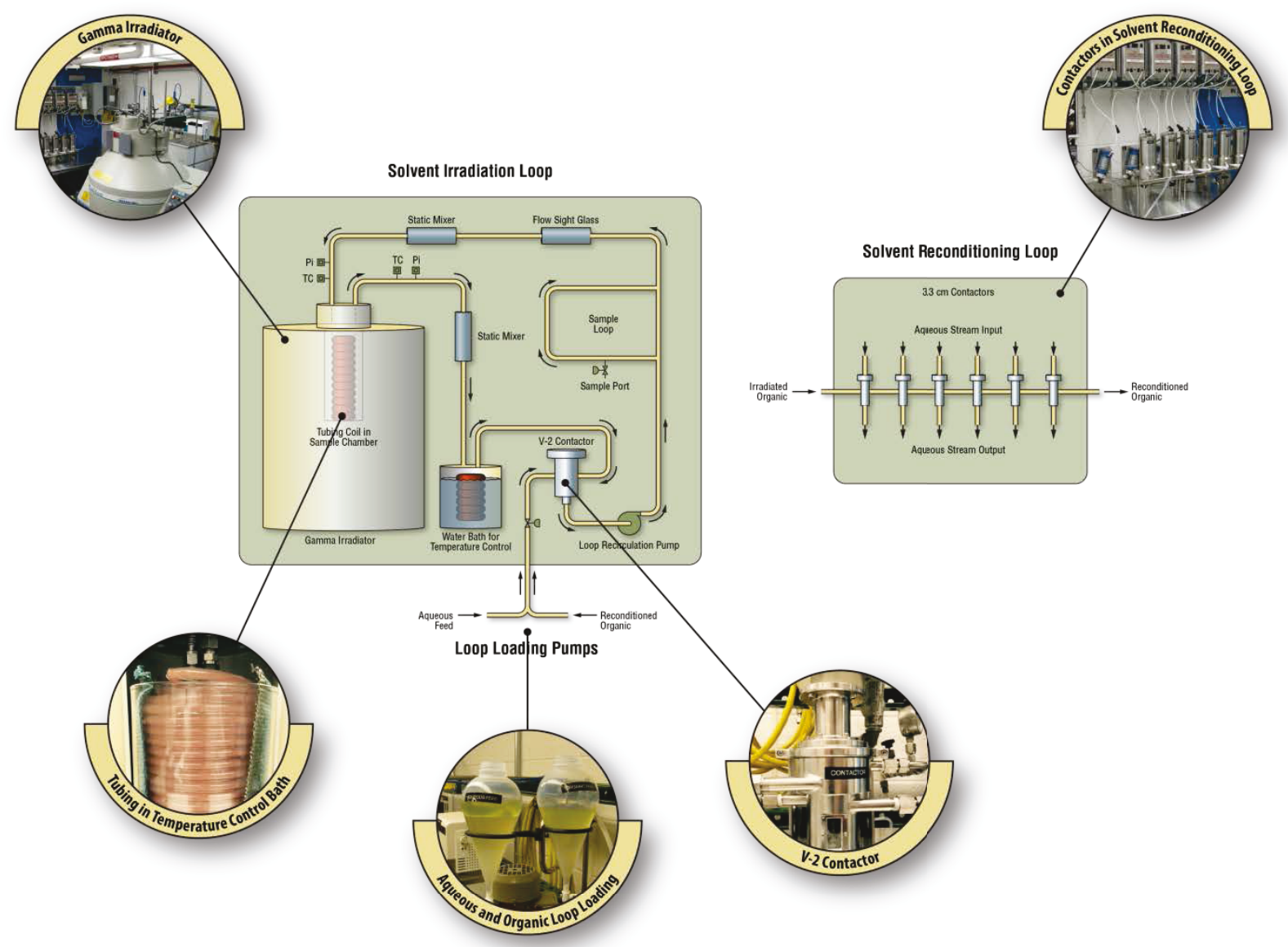

Figure 1. Schematic representation of solvent irradiation test loop.

The ALSEP process solvent uses a combination of the solvating extractant N,N,N',N'-tetra(2ethylhexyl)diglycolamide (T2EHDGA) and the cation exchanging extractant 2-ethylhexylphosphonic acid mono-2-ethylhexyl ester (HEH[EHP]) dissolved in dodecane. The actinides and lanthanides are coextracted from relatively high nitric acid and the actinides are selectively stripped using a carboxylic acid buffered aminopolycarboxylic acid solution. The structures of the compounds used in the ALSEP process organic phase are depicted in Figure 2. 


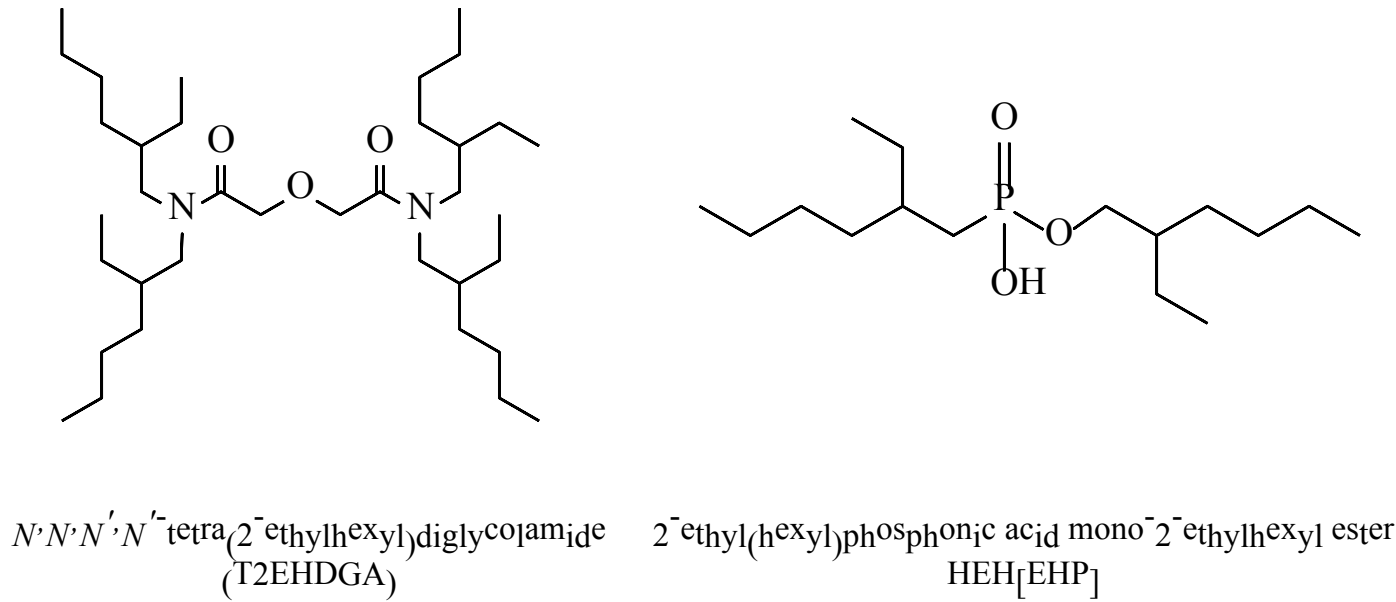

Figure 2. Structures of compounds used in the ALSEP process.

The FY-2015 experimental program at the INL focused upon examining two different ALSEP solvent compositions irradiated in contact with different aqueous phase acidities. The ALSEP solvent compositions studied contained 0.040 M T2EHDGA + 0.60 M HEH[EHP] dissolved in n-dodecane or $0.050 \mathrm{M}$ T2EHDGA + 0.75 M HEH[EHP] dissolved in n-dodecane; denoted ALSEP A and ALSEP B, respectively. A series of static irradiation experiments was designed in order to irradiate the ALSEP A and B solvent while in contact with either $2 \mathrm{M}$ or $4 \mathrm{M}$ nitric acid. During FY-2016, research efforts focused upon the analysis of these ALSEP static irradiations. The results of these analyses were used to finalize the aqueous and organic phase compositions which were utilized for the ALSEP test loop irradiation experiments performed in FY-2016. The original plan was to conduct one test loop experiment (ALSEP "B" in contact with $4 \mathrm{M}$ nitric acid); however, three additional tests were completed. The ALSEP test loop experiments performed were:

- Irradiate ALSEP " $\mathrm{B}$ " in contact with $4 \mathrm{M}$ nitric acid

- Irradiate ALSEP " $\mathrm{B}$ " in contact with $2 \mathrm{M}$ nitric acid

- Irradiate ALSEP "B" in contact with a scrub diluted feed

- ALSEP "B" $4 \mathrm{M}$ nitric acid hydrolysis.

For each of these test loop irradiations, the solvent systems were exposed to approximately 0.8 MGy total absorbed gamma dose which corresponds to 180 hours of test loop operation per test.

\section{RESULTS}

The impact of gamma radiolysis was evaluated by determining americium, europium, and cerium distribution ratios $\left(\mathrm{D}_{\mathrm{M}}=[\mathrm{Metal}]_{\mathrm{org}} /[\mathrm{Metal}]_{\mathrm{aq}}\right)$ for each solvent system as a function of absorbed gamma dose. Plots of the variation of determined values of $\mathrm{D}_{\mathrm{Am}}, \mathrm{D}_{\mathrm{Eu}}$, and $\mathrm{D}_{\mathrm{Ce}}$ as a function of absorbed dose for the $4 \mathrm{M}$ nitric acid, $2 \mathrm{M}$ nitric acid, and scrub diluted feed test loop experiments are shown in Figures 2 4, respectively. For each ALSEP "B" solvent system irradiated, gamma-irradiation does result in radiolytic degradation of the ALSEP solvent. However it should be noted, that the impacts of radiolytic degradation upon the efficacy of the ALSEP process appears to be relatively minor. 


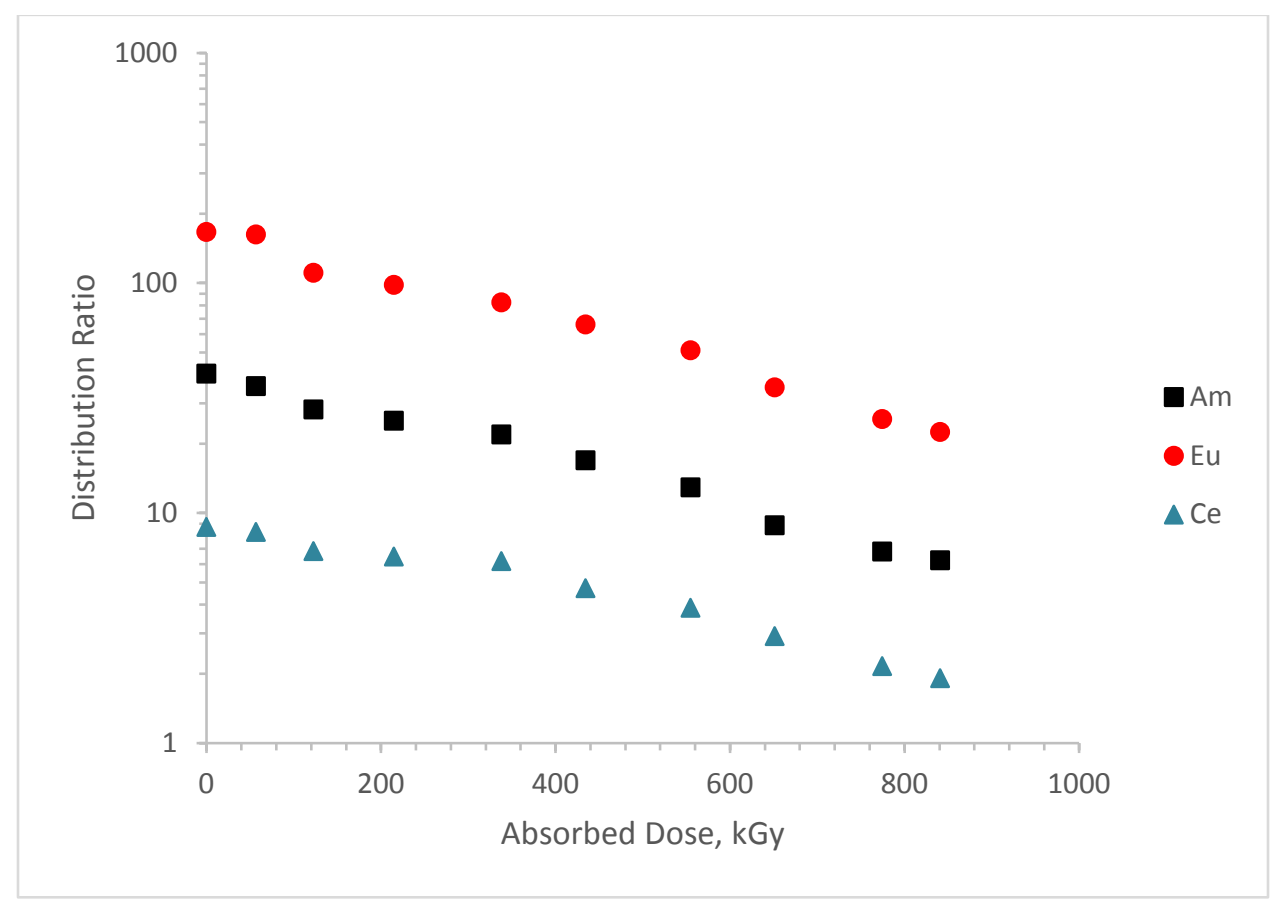

Figure 1. Values of $D_{A m}, D_{E u}$, and $D_{C e}$ determined as a function of absorbed dose for the test loop irradiation of $0.050 \mathrm{M}$ T2EHDGA / 0.75 M HEH[EHP] / n-dodecane (ALSEP "B") in contact with 4.0 M nitric acid.

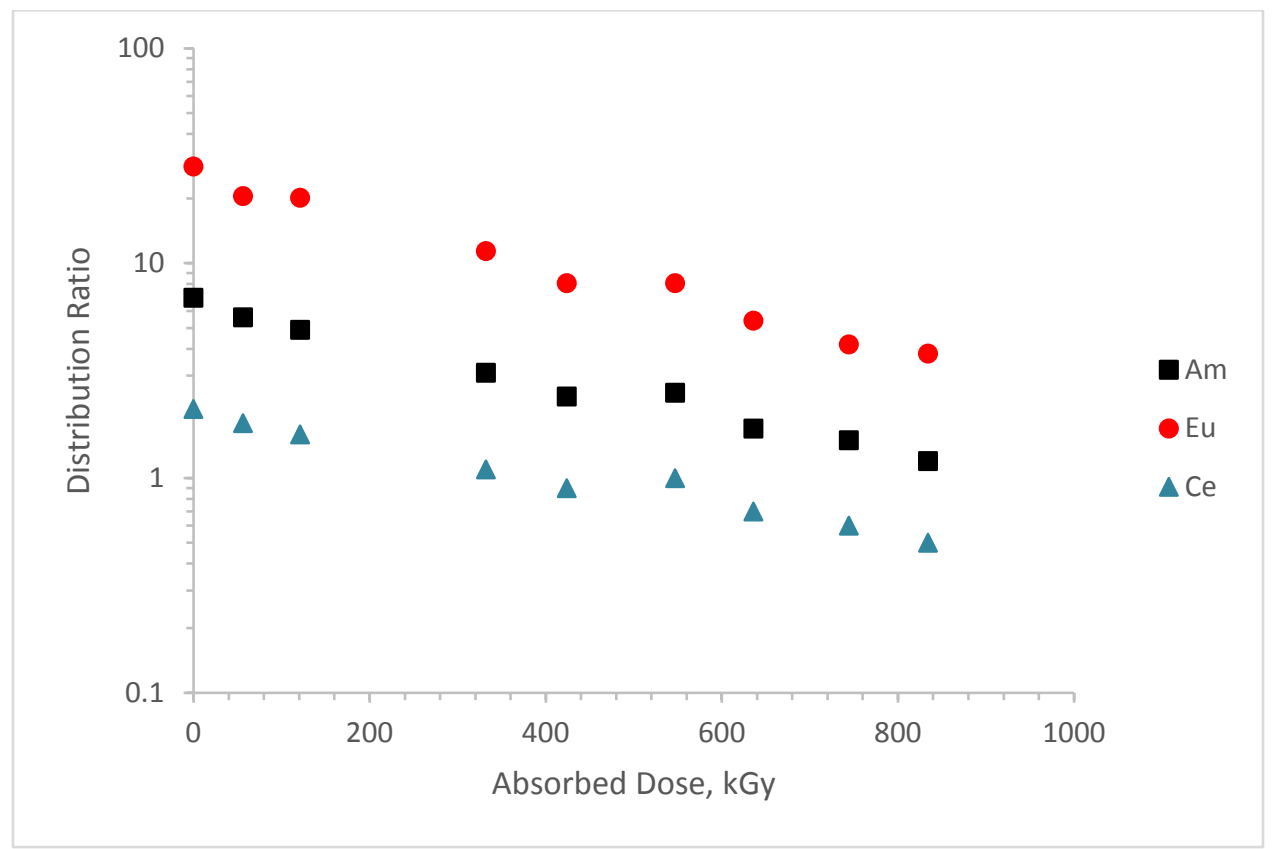

Figure 2. Values of $\mathrm{D}_{\mathrm{Am}}, \mathrm{D}_{\mathrm{Eu}}$, and $\mathrm{D}_{\mathrm{Ce}}$ determined as a function of absorbed dose for the test loop irradiation of 0.050 M T2EHDGA / 0.75 M HEH[EHP] / n-dodecane (ALSEP "B") in contact with $2.0 \mathrm{M}$ nitric acid. 


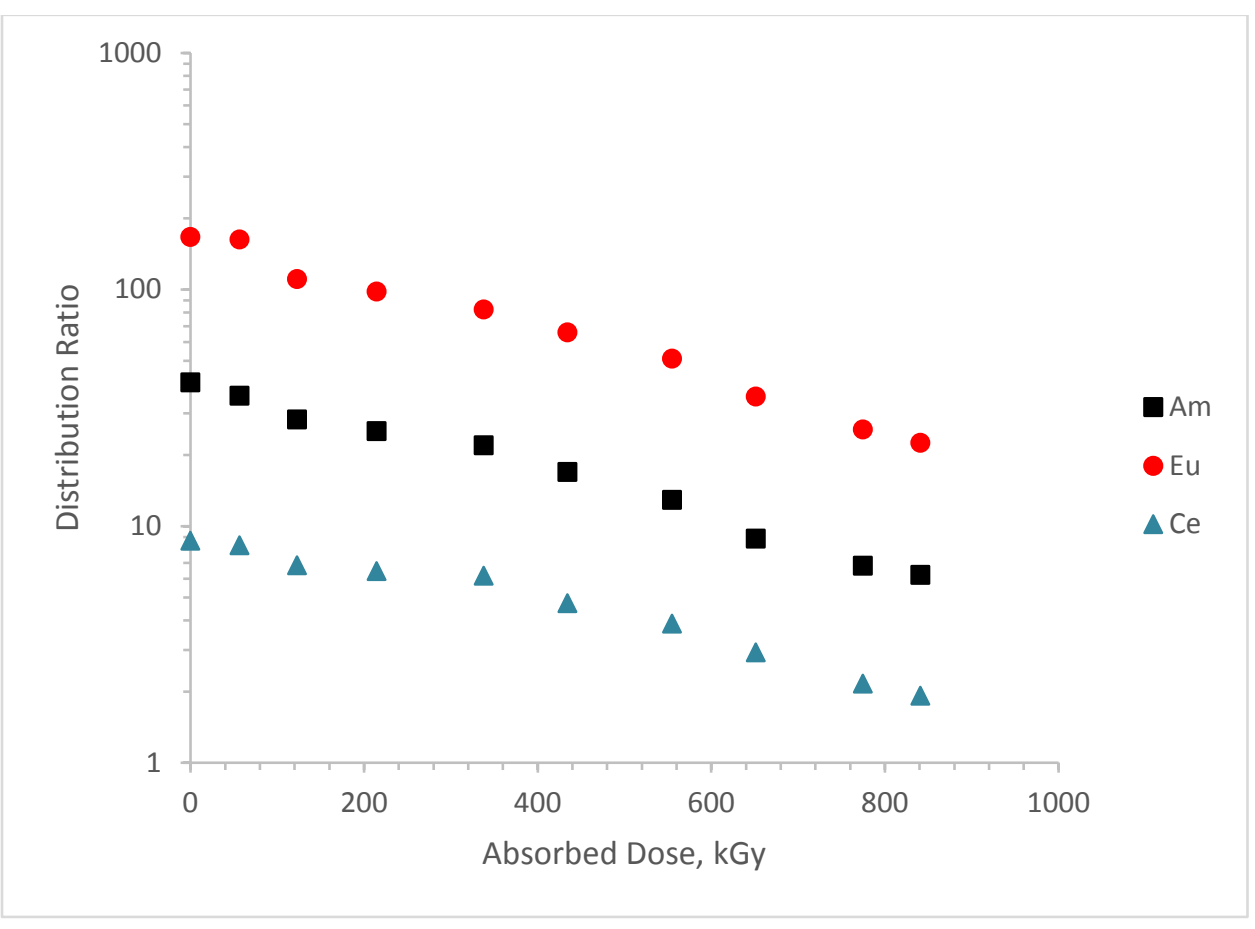

Figure 3. Values of $\mathrm{D}_{\mathrm{Am}}, \mathrm{D}_{\mathrm{Eu}}$, and $\mathrm{D}_{\mathrm{Ce}}$ determined as a function of absorbed dose for the test loop irradiation of 0.050 M T2EHDGA / 0.75 M HEH[EHP] / n-dodecane (ALSEP "B") in contact with a scrub diluted feed.

The possible impacts of hydrolytic degradation of the ALSEP solvent due to prolonged contact with nitric acid was evaluated by determining values of $D_{A m}, D_{E u}$, and $D_{C e}$ as a function of contact time with $4 \mathrm{M}$ nitric acid. This experiment was performed in the INL test loop without gamma irradiation. The values of the distribution ratios determined for the ALSEP hydrolysis test are presented in Figure 5. The highest contact time shown in Figure $5(\sim 185 \mathrm{hr})$ would correspond to an absorbed dose of $\sim 560 \mathrm{kGy}$. The distribution data presented in Figure 5 clearly demonstrate that hydrolytic degradation processes will not adversely impact the efficacy of the ALSEP solvent extraction process. 


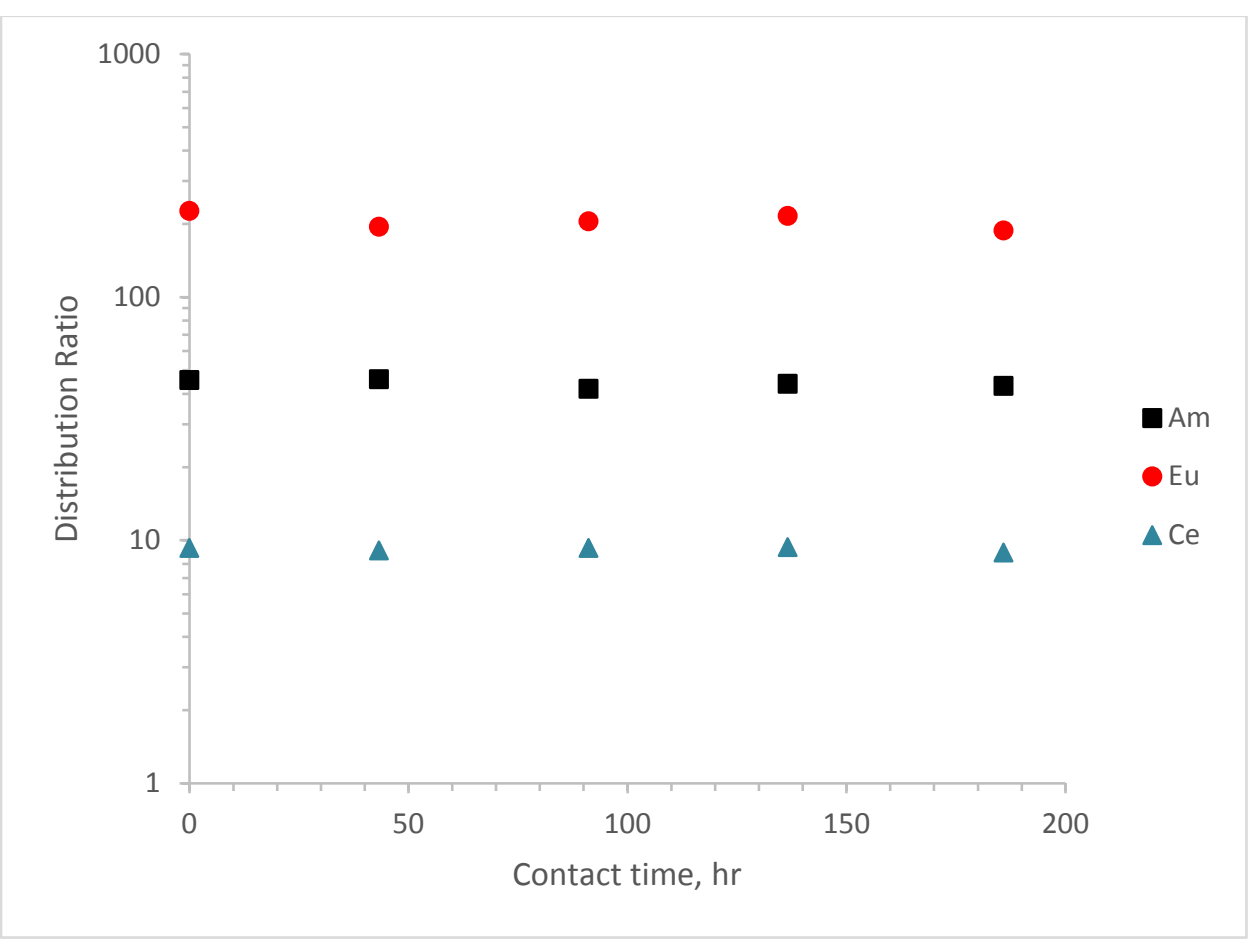

Figure 4. Values of $D_{A m}, D_{E u}$, and $D_{C e}$ determined as a function of contact time for $0.050 \mathrm{M}$ T2EHDGA / $0.75 \mathrm{M}$ HEH[EHP] / n-dodecane (ALSEP "B") in contact with $4 \mathrm{M}$ nitric acid.

\section{CONCLUSIONS}

Four ALSEP test loop experiments were performed: 1)ALSEP "B" in contact with $4 \mathrm{M}$ nitric acid, 2)ALSEP " $B$ " in contact with $2 \mathrm{M}$ nitric acid, 3)ALSEP " $\mathrm{B}$ " in contact with a scrub diluted feed, and 4)ALSEP "B" $4 \mathrm{M}$ nitric acid hydrolysis. For each of the test loop irradiations, the solvent systems were exposed to approximately 0.8 MGy total absorbed gamma dose. Distribution ratios as a function of adsorbed dose indicate gamma-irradiation does result in radiolytic degradation of the ALSEP solvent. However, it should be noted that the impacts of radiolytic degradation upon the efficacy of the ALSEP process appear to be relatively minor. For the hydrolysis test, the solvent was contacted with $4 \mathrm{M}$ nitric acid for about 200 hours with no observed impact to distribution rations. This demonstrates that hydrolytic degradation processes will not adversely impact the efficacy of the ALSEP solvent extraction process. 